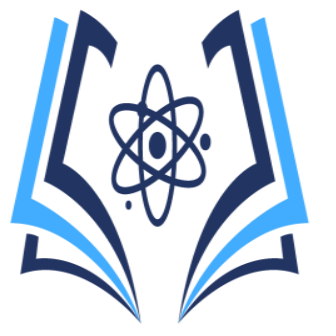

\title{
Determinantes sociales de salud y riesgos de padecer diabetes mellitus tipo 2
}

\section{Social determinants of health and risks of type 2 diabetes mellitus}

\section{Determinantes sociales de salud y riesgos}

Jorge Solórzano Segovia. Md. ${ }^{1}$

María Segovia Medina. Mg.GE. ${ }^{2}$

Maryuri Delgado Armijos, Mg.GE. ${ }^{3}$

Edwin Delgado Armijos. Biól.Pesc. ${ }^{4}$

${ }^{1}$ Unidad Médica JS Salud, Portoviejo, email: drjorgealbertosolorzanosegovia@gmail.com, Código Orcid: https://orcid.org/0000-0003-1020-0631

${ }^{2}$ Unidad educativa fiscal Portoviejo, email: marielenasegoviamedina@yahoo.com, Código Orcid: https://orcid.org/0000-0002-1979-6542

${ }^{3}$ Unidad educativa fiscal Abdón Calderón, Consejo Académico del Circuito, email: dmayito@yahoo.com, Código Orcid: https://orcid.org/0000-0003-1458-138X

4 Investigador Profesional independiente, email: mandela58@hotmail.com, código Orcid: https://orcid.org/0000-0002-86295262

Contacto: drjorgealbertosolorzanosegovia@gmail.com.

Recibido: 10-08-2020 Aprobado: 15-12-2020

\section{Resumen}

Objetivo. El objetivo de la investigación fue identificar las determinantes sociales en la salud y el riesgo de padecer diabetes mellitus tipo 2. Métodos. Se llevó a efecto un estudio observacional, transversal, retrospectivo de tipo descriptivo, a 60 pacientes entre los 30 y 75 años de edad, basado en los datos obtenidos en el Consultorio JS SALUD del cantón Montecristi, Manabí, Ecuador, desde mayo a agosto de 2020. En base a las variables género, ocupación laboral, ingresos económicos, factores educativos, factores de riesgo, antecedentes patológicos personales. Resultados. En base a los datos tabulados las determinantes sociales de salud hubo que predominar, factores educativos, ingresos económicos, ocupación laboral, factores de riesgos modificables. En cuanto a la diabetes mellitus tipo 2 se presentó en mayor porcentaje en hombres y tuvieron mayor índice de comorbilidades, la mayor comorbilidad acompañante de la DM2 es la hipertensión arterial. Conclusiones. La posición económica es un dato que se debe tener en consideración, por cuanto, a mayores ingresos las posibilidades de llevar una dieta es algo que se puede conseguir con facilidad. El consumo de carbohidratos es mayor entre las personas que conforman poseen niveles educativos bajos, sumado al hecho que entre los hábitos sociales incluyen consumo de alcohol de manera consuetudinaria, fumar cigarrillos. La diabetes mellitus tipo 2 es una patología que sigue ganando espacio, que en el caso de la población objeto de estudio asentada en Montecristi, por conocimiento del investigador, es elevada en consideración a la población total.

Palabras claves: Determinantes sociales de salud, diabetes mellitus tipo 2, hábitos sociales, escolaridad.

\begin{abstract}
Objective. The objective of the research was to study the social determinants of health and the risk of suffering from type 2 diabetes mellitus. Methods. An observational, cross-sectional, descriptive, retrospective study was carried out in
\end{abstract}


40 patients between 30 and 75 years of age, based on data obtained in the JS SALUD Clinic of the Montecristi canton, Manabí, Ecuador from May to August 2020 Based on the variables sex, family history, family pathological history, heart rate, respiratory rate. Results. Based on the tabulated data, the predominant social determinants of health were education, income, race, economic position, and social habits. Regarding type 2 diabetes mellitus, it was presented in a higher percentage in men and they had a higher rate of comorbidity, the greatest accompanying comorbidity of DM2 is arterial hypertension. Conclusions. The economic position is a fact that must be taken into consideration, since the higher the income the possibilities of following a diet are something that can be achieved easily. The consumption of carbohydrates is higher among the people who have low educational levels, added to the fact that among the social habits include the consumption of alcohol in a customary way, smoking cigarettes. Type 2 diabetes mellitus is a pathology that continues to gain space, which in the case of the population under study settled in Montecristi, according to the researcher's knowledge, is elevated in consideration of the total population.

Keywords: Social determinants of health, type 2 diabetes mellitus, social habits, schooling.

\section{Introducción}

La diabetes mellitus actualmente es considerada como enfermedad social, debido a la frecuencia elevada, también por los costos económicos que contempla a los gobiernos y familias de quienes padecen esta patología. El interés académico y científico relacionado con la calidad de vida y los aspectos psicosociales de las personas que poseen la diabetes mellitus tipo 2 ha ido en aumento. El estilo de vida causado por la modernidad, hábitos que implican el sedentarismo, comida rápida, desembocó en la epidemia actual de DM tipo 2 en el mundo.

El constante crecimiento de la prevalencia de diabetes mellitus tipo 2 es un problema de salud pública, una preocupación para los organismos nacionales e internacionales como la Organización Mundial de la Salud. Los determinantes sociales de salud como, ocupación laboral, ingresos económicos, posición económica, factores educativos, factores de riesgo modificable y no modificable, forman parte de una variable, Mientras que las comorbilidades: hipertensión arterial, neuropatía diabética, alergias, esteatosis hepática, por nombrar unos cuantos. A pesar se aquello, resulta necesario que se analice el problema con un enfoque holístico, desde perspectivas amplias, que permita tomar acciones tanto en el contexto de los hábitos sociales, la situación económica, culturales, educativa, con la finalidad de desarrollar propuestas para que sean aplicadas en el contexto local y nacional, como manera de frenar esta patología que conlleva problemas sanitarios, sociales y económicos.

\section{Determinantes del estado de salud}

La medicina incrementa continuamente su enfoque social, donde la familia, como célula fundamental de la sociedad, resulta un objeto de trabajo esencial para el equipo de salud, pues posee una incidencia importante en el desarrollo del proceso saludenfermedad. Sin embargo, los determinantes sociales en la salud indican la existencia de inequidades sanitarias, o sea, de las diferencias injustas y evitables observadas en este ámbito. A tales efectos, se exponen algunos aspectos relacionados con la repercusión de dichos determinantes sociales en el funcionamiento de la familia cubana y su salud.

En el conocimiento popular se inscribe la creencia de que la aparición de las enfermedades se relaciona directamente con la acción de causas biológicas, genéticas, contagiosas u orgánicas que ejercen tal influencia en el organismo humano que logran sobrepasar las barreras defensivas de este, desequilibran su funcionamiento $\mathrm{y}$ provocan malestares, síntomas y hasta la muerte (Berenguer, Pérez, Dávila y Sánchez, 2017).

De hecho, la concepción de salud como estado de bienestar físico, mental y social, como capacidad de funcionamiento y calidad de vida, y como conjunto de condiciones sociales digna y segura para la vida, son adquisiciones más recientes en la representación social de la salud.

El concepto de la salud como derecho humano fundamental, consignado en la Carta de Ottawa, destaca determinados prerrequisitos para la salud, que incluyen la paz, adecuados recursos económicos y alimenticios, vivienda, un ecosistema estable y un uso sostenible de los recursos (Vidal, Chamblas, Zavala, Müller, Cecilia y Chávez, 2014). El reconocimiento de estos prerrequisitos pone de manifiesto la estrecha relación que existe entre las condiciones sociales y 
económicas, el entorno físico, los estilos de vida individuales y la salud.

Estos vínculos constituyen la clave para una comprensión holística de la salud que es primordial en la definición de la promoción de la salud. Una visión integral de la salud supone que todos los sistemas y estructuras que rigen las condiciones sociales y económicas, al igual que el entorno físico, deben tener en cuenta las implicaciones y el impacto de sus actividades en la salud y el bienestar individual y colectivo

\section{Definición}

Los determinantes sociales de la salud (DSS) son las condiciones en las que las personas se desarrollan. Dichas condiciones impactan en sus vidas, condición de salud y nivel de inclusión social. Acorde a la evolución conceptual y de comprensión de la discapacidad, resulta importante actualizar los DSS por sus amplias implicancias en la ejecución de intervenciones de salud en la población.

Los DSS, según la Organización Mundial de la Salud, se definen como las circunstancias en que las personas nacen, crecen, viven, trabajan y envejecen, incluido el sistema de salud (Tamayo, Besoaín y Rebolledo, 2018). Esas circunstancias son el resultado de la distribución del dinero, el poder y los recursos a nivel mundial, nacional y local, que depende a su vez de las políticas adoptadas. Las principales propiedades que posee el modelo de los DSS es que cada factor es importante por sí mismo; están interrelacionados, actúan en distintos niveles, son acumulativos y son causales o protectores. Los DSS reflejan el estado de la salud como consecuencia de comportamientos individuales y contextos sociales, además del impacto de los servicios de salud y de las características genético-biológicas.

Los determinantes sociales de la salud que en su integralidad abordan elementos que intentan explicar que las desigualdades en que se manifiestan los resultados en salud para diversos grupos de la sociedad, dicen relación con las distintas posibilidades que tienen las personas de desarrollarse en la vida y gozar de buena salud. Esas desigualdades se observan en las condiciones de vida de la primera infancia, la escolarización, la naturaleza del empleo y las condiciones de trabajo, las características físicas del medio construido y la calidad del medio natural en que vive la población.
Según el carácter de esos entornos, las condiciones físicas, el apoyo psicosocial y los esquemas de conducta varían para cada grupo, haciéndolos más o menos vulnerables a la enfermedad. La estratificación social también crea disparidades en el acceso al sistema de salud y en su utilización, lo que da lugar a desigualdades en la promoción de la salud y el bienestar, la prevención de enfermedades y las posibilidades de restablecimiento y supervivencia tras una enfermedad.

Los factores de riesgo o determinantes son aquellas condiciones (estímulos, conductas, características personales y del entorno) que inciden en el estado de salud, incrementan la probabilidad de enfermar y facilitan las condiciones para su manifestación. Los determinantes del estado de salud han estado sometidos a las contingencias históricas de la especie humana. Dichos determinantes abarcan desde factores ambientales, biológicos, conductuales, sociales, económicos, laborales, culturales y, por supuesto los servicios sanitarios como respuesta organizada y especializada de la sociedad para prevenir la enfermedad y restaurar la salud (Vásquez, Macías, González, Pérez y Carrillo, 2019).

Al respecto, la medicina social y la salud colectiva surgieron del reconocimiento de que los procesos de salud/enfermedad, las representaciones de estos y las respuestas sociales para enfrentarlos, expresan hechos histórico-sociales que atañen a los colectivos humanos; por tanto, es necesario explicar la determinación y distribución de estos procesos más allá de su causalidad próxima y del ámbito de la biología.

Según el concepto de la Organización Mundial de la Salud (OMS), los determinantes son un conjunto de factores personales, sociales, económicos y ambientales que determinan el estado de salud de los individuos o las poblaciones. Comprenden los comportamientos y los estilos de vida saludables, los ingresos y la posición social, la educación, el trabajo y las condiciones laborales, el acceso a servicios sanitarios adecuados y los entornos físicos. Combinados todos ellos, crean distintas condiciones de vida que ejercen un claro impacto sobre la salud (Berenguer, Pérez, Dávila y Sánchez, 2017).

\section{Importancia de destacar los determinantes sociales de la salud.}


$>$ Los determinantes sociales de la salud repercuten directamente en la salud de las familias.

$>$ Los determinantes sociales de la salud permiten predecir la mayor proporción de la variación del estado de salud.

$>$ Los determinantes sociales de la salud estructuran los compartimientos relacionados con la salud.

D Los determinantes sociales de la salud interactúan mutuamente en la generación de salud (figura).

\section{Clasificación de las determinantes del estado de salud}

Los determinantes sociales de la salud han permitido entender las diferencias en salud cuyo origen va más allá de la biología, siendo de gran ayuda en la definición de políticas de salud pública, los múltiples DSS identificados pueden ser clasificados siguiendo diversos criterios, entre los cuales destaca el Rainbow Model de Dahlgren y Whitehead. Este modelo propone tres posibles niveles dependiendo de si se trata de características propias de las personas (micro) o del contexto en el que se localizan las interacciones de diversas personas (mezzo en el caso del contexto más cercano -familia, lugar de residencia, entre otros, y, macro en el contexto más general -políticas públicas, características socioculturales, medios de comunicación, entre otros.

\section{Relación entre salud y nivel educativo}

La asociación entre salud y educación ha sido probada por la capacidad de esta última para definir los niveles de estratificación social, aquellos grupos poblacionales definidos por su bajo nivel educativo muestran una mayor desventaja en salud, aunque con diferencias entre países debido a sus especificidades en cuanto a comportamientos saludables o políticas públicas. Por ejemplo, en España, a pesar de la progresiva mejora de la salud autopercibida en las recientes décadas, la población con menor nivel educativo ha seguido declarando una peor salud. En el mismo sentido, se ha encontrado datos que indican que la población española con menor nivel educativo mostraba peores valores para los indicadores de estilo de vida, como consumo de tabaco, alcohol y sedentarismo, además de mayores problemas para llevar a cabo las actividades diarias.

\section{Relación entre salud y el hogar}

El hogar como DSS se ubica en el nivel intermedio al entenderse como el contexto común de convivencia de personas emparentadas, entre las que se establece un intercambio continuo y diario de recursos de naturaleza diversa (económicos, emocionales, de cuidado, entre otros. En este sentido, en general, la convivencia en pareja ha mostrado asociarse a un mejor perfil de salud respecto a la población homóloga que no vive en pareja. Esta diferencia ha sido justificada por la optimización de los recursos mediante economías de escala dentro del contexto de la pareja, o por la creación y el mantenimiento de una mayor red social que puede ser de ayuda ante posibles inconvenientes (Gumà, Arpino y Solé, 2017).

Las determinantes sociales de la salud surgen con fuerza desde hace aproximadamente dos décadas, ante el reconocimiento de las limitaciones de las intervenciones dirigidas a los riesgos individuales de enfermar y que no tomaban en cuenta el rol de la sociedad. La mujer en edad mediana transita de la vida reproductiva a la no reproductiva. Se inicia alrededor de los 40 años, con el proceso premenopausia, menopausia y climaterio; finalizando al comenzar la senectud aproximadamente a los 59 años de edad. Esta etapa de la vida presenta características específicas en condiciones sociales que afectan a la salud.

Las determinantes sociales surgen en la década de los noventa como un nuevo concepto: Es la percepción que tiene el individuo de los efectos de una enfermedad o de la aplicación de un tratamiento sobre su bienestar físico, emocional o social. Esta perspectiva nos permite apreciar la percepción que tienen las personas sobre su salud, en este caso la mujer en edad mediana. El estudio de las determinantes sociales viene a constituir un nuevo indicador para la valoración de las consecuencias de las enfermedades sobre la percepción de salud, así como de los resultados de las diferentes intervenciones médicas en el manejo del proceso salud-enfermedad (González, Bayarre y Hernández, 2019).

En relación a las DSS el trabajo más reciente y al que se le ha dado mayor difusión provino de la OMS, que, al analizar el impacto favorable que tenían las acciones dirigidas a disminuir el efecto 
de algunos de los DSS en estudios realizados en distintos países (Jasso y López, 2014).

En términos generales, se dividió a los DSS en estructurales e intermediarios. Con respecto a los primeros, se reconoce como punto de partida a nivel macro un amplio contexto socioeconómico y político, mientras que a nivel micro o individual se incluyen los factores educativos, empleo $\mathrm{u}$ ocupación e ingreso, entre otros. Por su parte, los determinantes intermediarios incluyen los factores biológicos, estilo de vida, condiciones de vivienda y al sistema de salud encargado de brindar esos servicios (Tamayo, Besoaín y Rebolledo, 2018).

\section{Diabetes mellitus tipo 2}

La diabetes mellitus tipo 2 (DM 2) es una destrucción progresiva de las células beta mediada por factores metabólicos genéticamente determinados junto con el aumento de factores inflamatorios (interleucinas) que inducen apoptosis y disminución del volumen de las células beta. Inicialmente se produce un aumento compensador de la secreción de insulina (hiperinsulinemia) es decir existirá una mayor entrega de insulina luego veremos una menor entrega de insulina (hipoinsulinemia) y aquí se da una intolerancia a la glucosa el cual se desencadenará en la diabetes por la destrucción de la célula beta. Las personas con diabetes mellitus tipo 2 (DM2) a menudo pueden tratar inicialmente su afección mediante ejercicio y dieta. Sin embargo, con el tiempo la mayoría de las personas requieren medicación oral o insulina.

La diabetes mellitus tipo 2(DMII) es una afección ocasionada por múltiples causas, donde los determinantes sociales de la salud descritos anteriormente están frecuentemente relacionados y todo lo cual conlleva a pensar, cada vez con más evidencia, que esta es una enfermedad social (8). En la actualidad la Diabetes es una de las principales causas de morbimortalidad en muchos países de América latina. Además, los pacientes diabéticos sufren cardiopatías y otras complicaciones si se comparan con la población no diabética.

La diabetes mellitus es una enfermedad crónica, degenerativa, que requiere un continuo cuidado médico y educación del paciente, para obtener así un correcto control de la glicemia. Dentro de las principales complicaciones de la misma, se encuentra la microangiopatía que afecta a riñones, extremidades inferiores y globos oculares. La retinopatía diabética genera una disminución progresiva de la agudeza visual que, a largo plazo, puede producir ceguera.

La OMS, afirma que existen en la actualidad, 422 millones de personas con diabetes a nivel mundial y se estima que la diabetes será la séptima causa de mortalidad en 2030. En América se estima que existirán 64 millones, $17 \%$ no diagnosticados y podrían incrementarse en un 55\% en el 2035.

La diabetes mellitus (DM) trata de un grupo de trastornos metabólicos caracterizado por niveles altos de glucosa en sangre, mientas que la DM tipo 2 es un grupo heterogéneo de trastornos que se caracteriza por grados variables de resistencia a la insulina, alteraciones tanto en la secreción de la insulina, como en la producción excesiva de glucosa hepática.

La OMS estimó que 347 millones de personas estaban afectadas de diabetes en 2014 en el mundo, y se prevé que para 2030, esta enfermedad constituya la séptima causa mundial de muerte (Llorente, Miguel, Rivas y Borrego, 2016). Los factores de riesgo asociados a DM 2 comprenden, entre otros, antecedentes familiares de diabetes, hipertensión arterial (HTA), dislipidemias y obesidad, cuya identificación en los pacientes afectados permitiría la prevención o reducción de sus complicaciones a corto y largo plazo, con el diseño e implementación de estrategias de intervención y campañas educativas que modifiquen esos factores, como se ha demostrado en diversos estudios

Por ser una enfermedad crónica de alta prevalencia con complicaciones de importante repercusión en la salud de los pacientes diabéticos, genera que la inversión en su tratamiento se incremente. A nivel mundial, afecta aproximadamente a 130 millones de personas, y se estima que la cifra llegará a 300 millones para el año 2025. En la mayoría de los países, casi el $50 \%$ de los pacientes desconocen su enfermedad, por tanto, no reciben tratamiento; un 20 a $30 \%$ de los pacientes que conocen su enfermedad no cumplen ningún tipo de tratamiento y el $68 \%$ de los casos de DM conocen su diagnóstico a consecuencia de la manifestación clínica de complicaciones.

La diabetes significa que la glucosa en la sangre, también llamada azúcar en la sangre, está muy alta. Con la diabetes tipo 2, la más común, el cuerpo no produce o no usa bien la insulina. La insulina es una 
hormona que ayuda a la glucosa a entrar a las células para darles energía. Sin insulina hay demasiada glucosa en la sangre. Con el tiempo, un nivel alto de glucosa en la sangre puede causar problemas serios en el corazón, los ojos, los riñones, los nervios, las encías y los dientes.

La persona presenta un riesgo mayor de tener diabetes tipo 2 si es adulto mayor, tiene obesidad, historia familiar de diabetes o no hace ejercicio. Sufrir de prediabetes también aumenta ese riesgo. Las personas que tienen prediabetes tienen un nivel de azúcar más alto del normal pero no lo suficiente como para ser considerados diabéticos. Si está en riesgo de diabetes tipo 2 , podría retrasarla o prevenir su desarrollo al hacer cambios en su estilo de vida.

\section{Síntomas de la DM2}

Los síntomas de la diabetes tipo 2 aparecen lentamente. Algunas personas ni siquiera los notan. Pueden incluir

$>$ Mucha sed
$>$ Micción frecuente
$>$ Sentirse hambriento o cansado
$>$ Perder peso sin proponérselo
$>$ Tener heridas que sanan lentamente
$>$ Visión borrosa

Un tipo de prueba, la A1c, también puede comprobar cómo está manejando su diabetes. Muchas personas controlan su diabetes a través de una alimentación saludable, actividad física y exámenes de glucosa en la sangre.

Además, en la actualidad existen estudios que demostraron que los cambios en estilos de vida hacen que los individuos sean más propensos a desarrollar DM II, debido a los cambios progresivos y acelerados. Por este motivo, se sospecha que puede afectar la incidencia de la patología para el caso de Latinoamérica y países andinos como: Perú, Bolivia, Ecuador y Guatemala, donde el $40 \%$ de la población son indígenas y entre el $20 \%$ y el $40 \%$ de ellos son habitantes rurales. Se estimó que la prevalencia de DM II en zonas urbanas oscila entre 7 y $8 \%$, mientras. en las zonas rurales es apenas del 1 y $2 \%$ de los indígenas adultos mayores de 35 años que padecen DM II (Nivia, Romero, Dimaté y Rodríguez, 2016).
Ecuador no escapa a esta situación: En territorio ecuatoriano, la DM2 fue la primera causa de mortalidad para el año 2013, correspondiendo a 4695 muertes, 7,44\% del total anual. La rápida evolución de este patrón epidemiológico se vincula estrechamente con el auge de las enfermedades crónicas degenerativas en los países en vías de desarrollo, en el marco del fenómeno de transición epidemiológica (Altamirano, Vásquez, Cordero, Álvarez, Añez, Rojas y Bermúdez, 2017).

Hay varios factores posibles en el desarrollo de DM2, los cuales pueden clasificarse en factores modificables: obesidad, dieta hipercalórica, tabaquismo y falta de actividad física; y no modificables: edad avanzada, antecedente familiar de diabetes de primer grado, origen étnico y antecedentes gestacionales (Lovera, Castillo, Malarczuk, Castro, Bonneau, Ceballos, Sánchez, Jiménez y Vacchino, 2014).

La diabetes mellitus por su elevada morbimortalidad se encuentra entre las cuatro principales enfermedades no transmisibles que han devenido como uno de los mayores desafíos para el desarrollo del siglo XXI. Clásicamente la diabetes mellitus tipo 2 (DM2) ha sido considerada una condición de los adultos, pero las evidencias demuestran que tiene una incidencia creciente en niños y adolescentes (Valdés, Almirall y Gutiérrez, 2019).

La Organización Mundial de la Salud (OMS) ha pronosticado que en el año 2030 esta enfermedad afectará a 370 millones de personas, lo que supone un aumento de un $114 \%$ a nivel mundial con respecto al año 2000; se estima que el continente americano ocupa el tercer lugar en incidencia de esta enfermedad, y que en México se sitúa como la $7^{\mathrm{a}}$ causa de muerte (Sarabia, Can y Guerrero, 2016).

\section{Materiales y métodos}

Se efectuó un estudio observacional, transversal, retrospectivo de tipo descriptivo, a 50 pacientes en edades comprendidas entre 30 y 75 años de edad, que son atendidos en el Consultorio particular JS SALUD del cantón Montecristi, Manabí, Ecuador desde mayo a agosto de 2020. Se apoyó en las fichas clínicas de los pacientes, dichos datos se registraron en base a categorías como: género, antecedentes familiares, antecedentes patológicos familiares, frecuencia cardiaca, frecuencia respiratoria, posición económica, ingresos 
económicos, factores educativos, factores de riesgo modificables y no modificables que permitan la tabulación con la finalidad de contar con datos certeros. El muestreo fue aleatorio.

La revisión de la literatura de libros físicos, artículos científicos de Scielo, Redalyc, tesis y demás documentos de contenido científico en un período comprendido desde 2012 hasta 2019 permitió revisar los documentos de autores que investigaron en torno a las variables que forman parte del problema identificado. Las palabras claves utilizadas fueron: determinantes sociales en la salud, diabetes mellitus tipo 2, factores de riesgo, salud, nivel educativo, hogar, personas.

\section{Resultados}

\section{Determinantes sociales de la salud}

\begin{tabular}{lcc}
\hline Alternativas & Frecuencia & Porcentaje \\
\hline Ocupación laboral & 16 & $27 \%$ \\
\hline Ingresos económicos & 17 & $28 \%$ \\
\hline Factores educativos & 8 & $14 \%$ \\
\hline Factores de riesgo modificable & 11 & $18 \%$ \\
\hline Factores de riesgo no modificable & 8 & $13 \%$ \\
\hline Total & $\mathbf{6 0}$ & $\mathbf{1 0 0 \%}$ \\
\hline
\end{tabular}
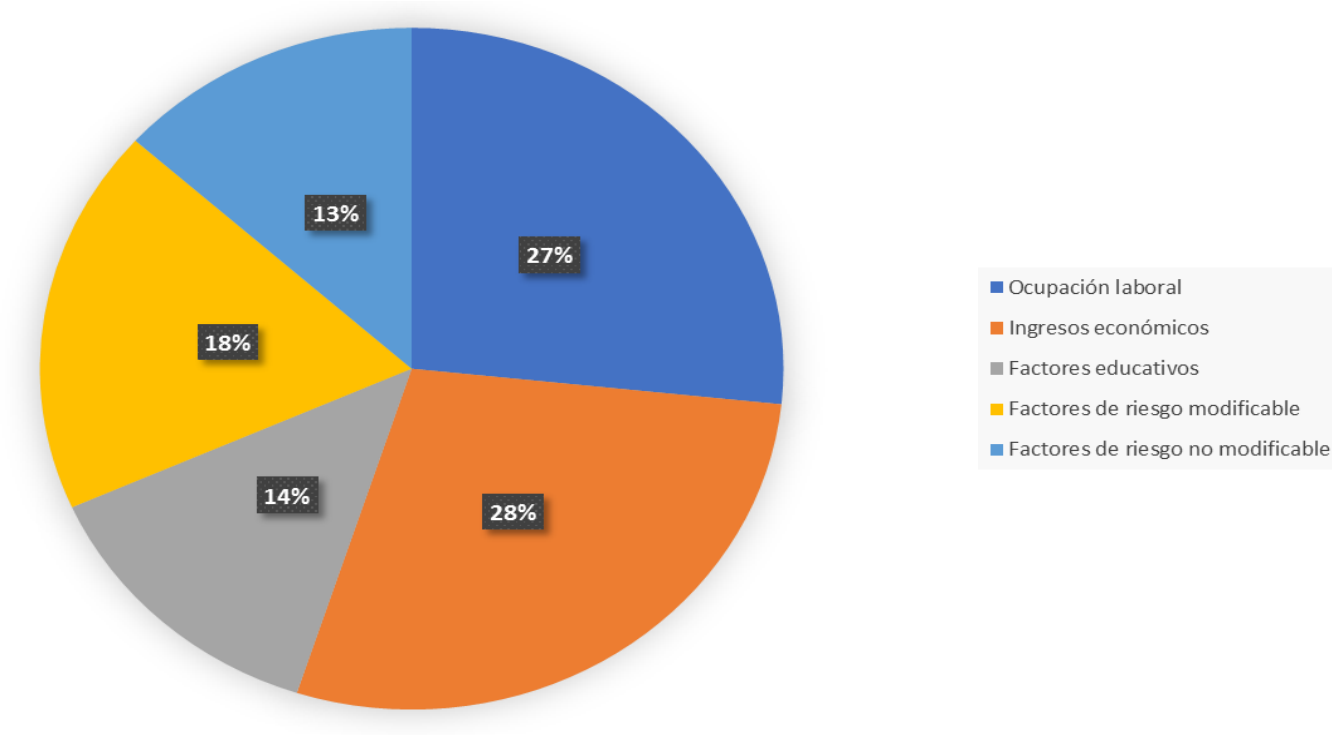

\begin{tabular}{lcc}
\hline Antecedentes patológicos personales & Hombres & Mujeres \\
\hline Hipertensión arterial & 8 & 10 \\
\hline Diabetes mellitus 2 & 19 & 12 \\
\hline Neuropatía diabética & 0 & 2 \\
\hline Alergias & 4 & 1 \\
\hline Esteatosis hepática & 0 & 1 \\
\hline Hipotiroidismo & 0 & 1 \\
\hline Evento cerebro vascular & 1 & 0
\end{tabular}




Hipoglicemia $\quad 0 \quad 0 \quad 1$

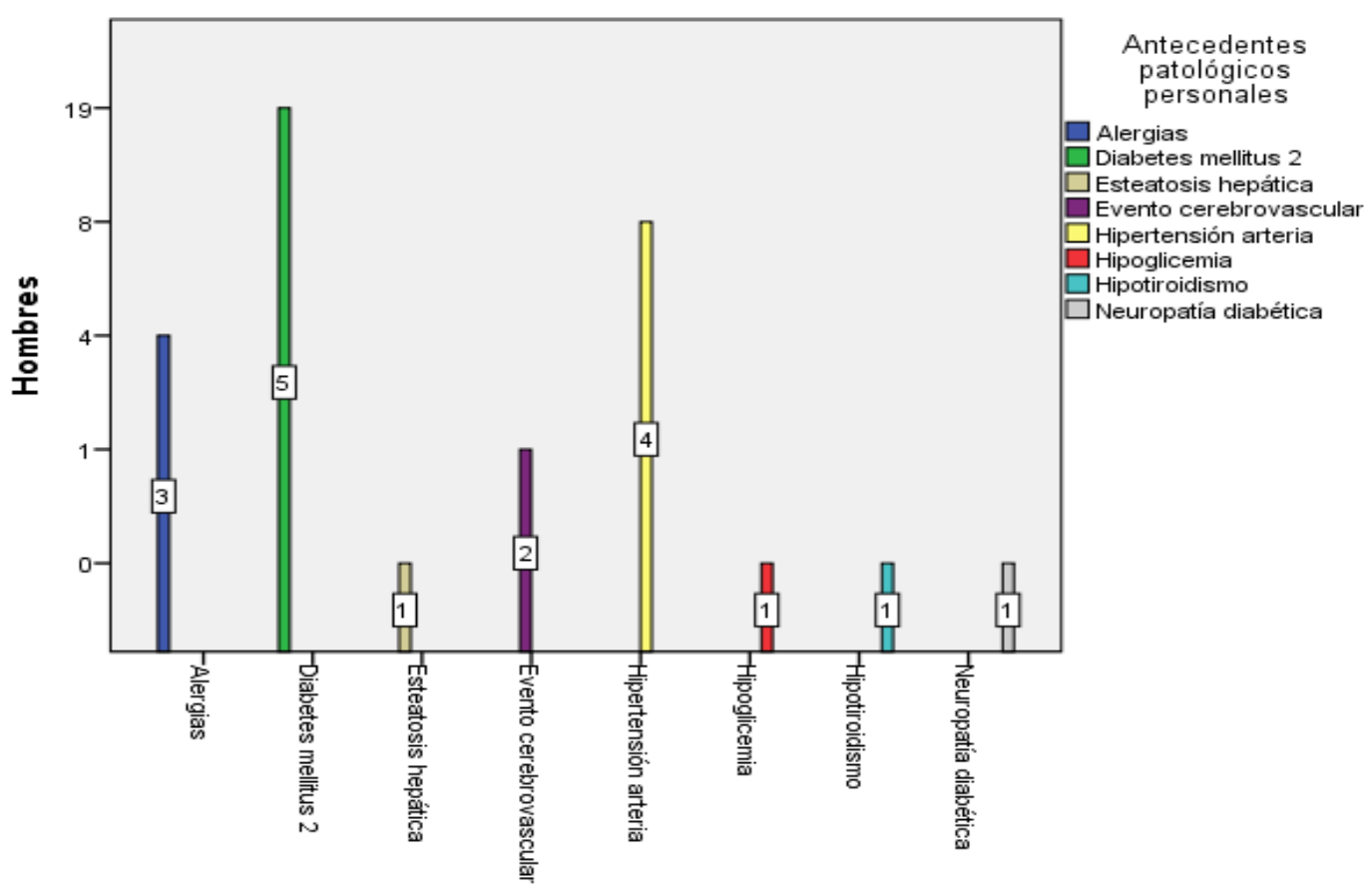

Antecedentes patológicos personales

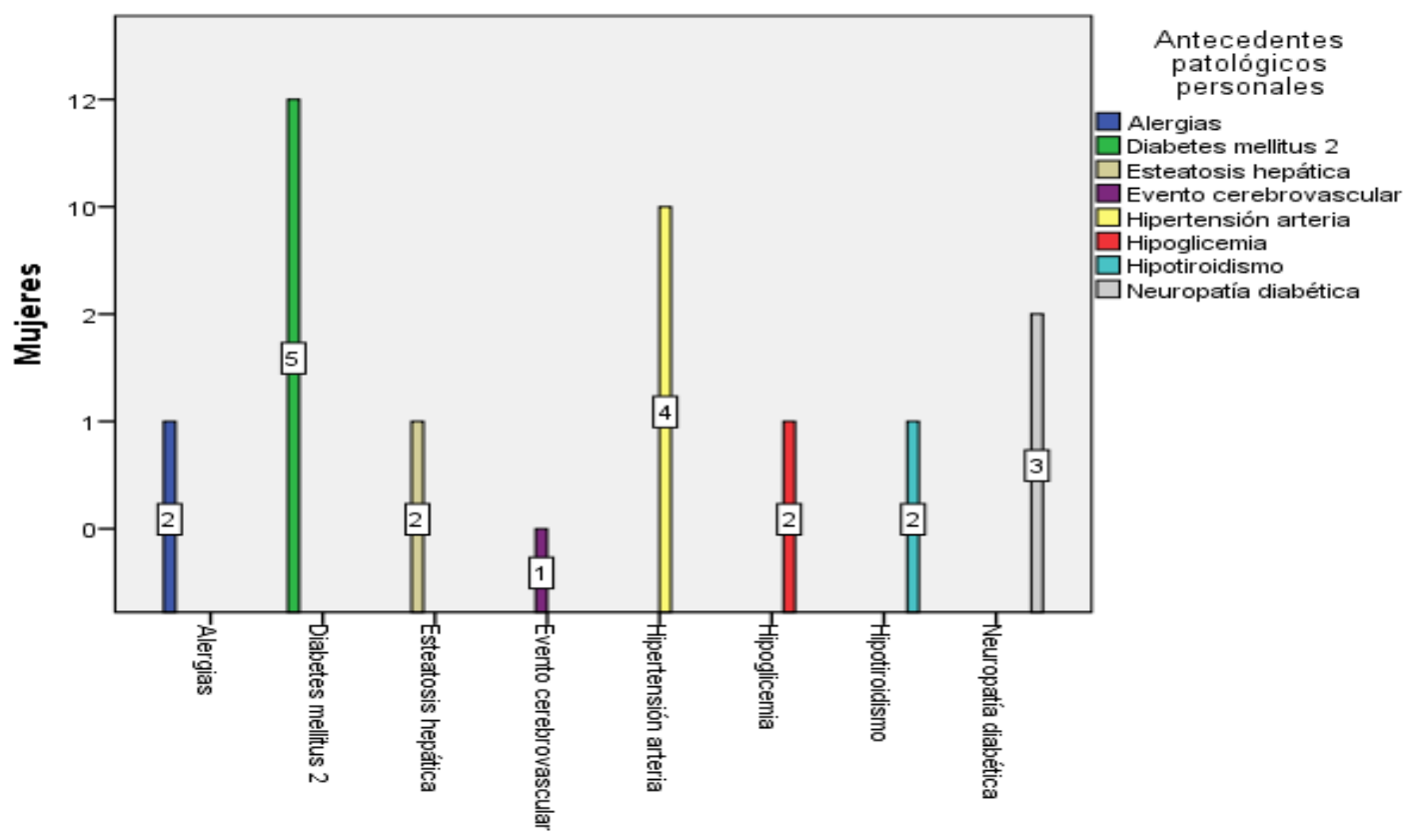

Antecedentes patológicos personales

https://revistas.itsup.edu.ec/index.php/higia 


\begin{tabular}{ccc}
\hline Patologías personales & Frecuencia & \% \\
\hline Hombres & 32 & $53 \%$ \\
\hline Mujeres & 28 & $47 \%$ \\
\hline Total & $\mathbf{6 0}$ & $\mathbf{1 0 0 \%}$
\end{tabular}

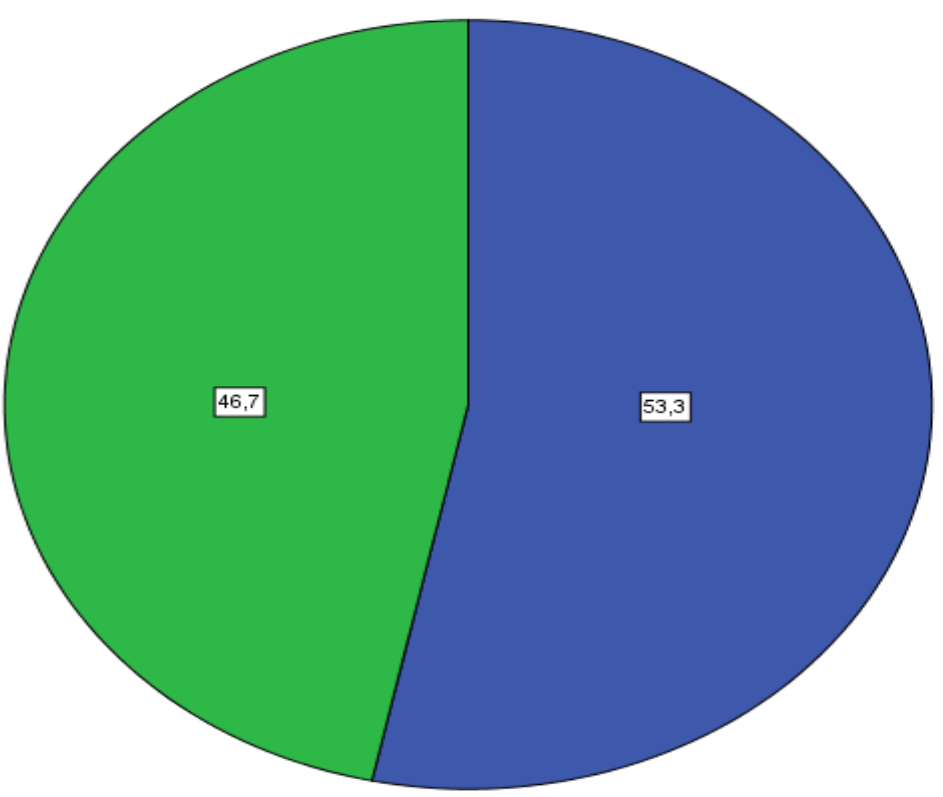

Patologías

personales

口ijeres

La investigación de campo permitió obtener datos que fueron tabulados para tener conocimiento acerca de las variables objeto de estudio, como es el caso de as determinantes sociales de salud, entre las cuales sobresalieron escolaridad, posición económica, ingresos económicos, hábitos sociales, ente otros. La diabetes mellitus tipo 2 tiene presencia tanto en hombres como en mujeres, sin embargo, para el presente estudio fue prevalente en los hombres, la comorbilidad acompañante de la DM2 que predominó fue la hipertensión arterial.

\section{Discusión}

Para que haya salud deben darse una serie de factores, confluencia de múltiples determinantes, ente ellos factores sociales, económicos, hábitos sociales, culturales, educativos, que terminan convergiendo para llegar a la consecución de la salud sistémica. Con mucha recurrencia los estudios efectuados sobre diabetes mellitus tipo 2 solo se fijan en los factores biológicos. A modo de ejemplo se cita el estudio desarrollado por Aguilar y Maldonado (2016) donde estableció en su investigación la no existencia de complicaciones entre la DM2 y los factores socioeconómicos.
Algo que se contradice con los datos obtenidos en el presente estudio. Sin embargo, Baquero, Mora y Rada (2019) sostienen que, en el contexto del estilo de vida integra el conjunto de conductas y hábitos personales en los seres humanos, que mantienen una alimentación equilibrada, abstinencia, o moderación, en el consumo de sustancias nocivas, y la práctica regular de actividad física, es decir, es el conjunto de factores dentro de los cuales se aprecian las determinantes sociales de salud.

\section{Conclusiones}

Los determinantes sociales de salud en la actualidad están siendo tomados en consideración, debido que se enfoca en la raíz del problema, en las causas que motivan al aparecimiento de la diabetes mellitus tipo 2. La posición económica es un factor clave que se debe tomar en consideración, por cuanto, a mayores ingresos las posibilidades de llevar una dieta es algo que se puede conseguir con facilidad.

El consumo de carbohidratos es mucho mayor entre personas que tienen niveles educativos bajos, además, los hábitos sociales que presenta la 
población estudiada incluyen consumo de alcohol, fumar cigarrillos, otros. La diabetes mellitus tipo 2 es una enfermedad que causa daños a la salud sistémica de quien la padece, la afectación es total a la economía humana.

\section{Bibliografía}

Aguilar, M. Maldonado, Y. (2016). Factores determinantes en las complicaciones de Diabetes Mellitus tipo 2 (DM 2) en adultos y adultos mayores atendidos en el Centro de Atención Primaria (CAP) III Es Salud - El Agustino, 2016. https://revistas.upeu.edu.pe/index.php/r_nutricion/article/view/943/Factores

Altamirano, L. Vásquez, M. Cordero, G. Álvarez, R. Añez, R. Rojas, J. Bermúdez, V. (2017). Prevalencia de la diabetes mellitus tipo 2 y sus factores de riesgo en individuos adultos de la ciudad de CuencaEcuador. Avances en Biomedicina, vol. 6, núm. 1, abril, 2017, pp. 10-21 Universidad de los Andes Mérida, Venezuela. https://www.redalyc.org/pdf/3313/331351068003.pdf

Baquero, D. Mora, M. Rada, L. Jiménez, W. (2019). Determinantes sociales de la salud influyentes en la complicación de pacientes hipertensos y diabéticos que padecen insuficiencia renal. Rev. Méd. Risaralda 2019; 25 (1) 15-21. http://www.scielo.org.co/pdf/rmri/v25n1/0122-0667-rmri-25-01-15.pdf

Berenguer, M. Pérez, A. Dávila, M. Sánchez, I. (2017). Determinantes sociales en la salud de la familia cubana. MEDISAN 2017; 21(1):61. http://scielo.sld.cu/pdf/san/v21n1/san08211.pdf

González, L. Bayarre, H. Hernández, E. (2019). Influencia de las determinantes sociales de salud en la calidad de vida relacionada con la salud de la mujer durante el envejecimiento. Archivos en Medicina Familiar. Vol.21 (1)1-5. 2019. https://www.medigraphic.com/pdfs/medfam/amf-2019/amf191a.pdf

Gumà, J. Arpino, B. Solé, A. (2017). Determinantes sociales de la salud de distintos niveles por género: educación y hogar en España. Gac Sanit 33 (2) Mar-Apr 201905 Dic 2019 • https://doi.org/10.1016/j.gaceta.2017.11.010. https://www.scielosp.org/article/gs/2019.v33n2/127$133 /$

Jasso, L. López, M. (2014). El impacto de los Determinantes Sociales de la Salud en los niños. Bol. Med. Hosp. $\begin{array}{llllll}\text { Infant. } & \text { Mex. } & \text { vol.71 no.2 } & \text { México } & & \end{array}$ http://www.scielo.org.mx/scielo.php?script=sci_arttext\&pid=S1665-11462014000200009

Llorente, Y. Miguel, P. Rivas, D. Borrego, Y. (2016). Factores de riesgo asociados con la aparición de diabetes mellitus tipo 2 en personas adultas. Rev Cubana Endocrinol vol.27 no. 2 Ciudad de la Habana mayo.ago. 2016. http://scielo.sld.cu/scielo.php?script=sci_arttext\&pid=S1561-29532016000200002

Lovera, M. Castillo, M. Malarczuk, C. Castro, C. Bonneau, G. Ceballos, B. Sánchez, A. Jiménez, S. Vacchino, M. (2014). Incidencia de Diabetes Mellitus tipo 2 y factores de riesgo en una cohorte de trabajadores de la salud. Acta Bioquímica Clínica Latinoamericana, vol. 48, núm. 1, marzo, 2014, pp. 45-52 Federación Bioquímica de la Provincia de Buenos Aires Buenos Aires, Argentina. https://www.redalyc.org/articulo.oa?id=53531786007

Nivia, G. Romero, U. Dimaté, A. Rodríguez, D. (2016). Factores de riesgo asociados a la diabetes mellitus tipo ii en indígenas de Latinoamérica. Revisión de literatura 2000-2016.

Sarabia, B. Can, A. Guerrero, J. (2016). Identificación de Factores de Riesgo de la Diabetes Mellitus Tipo 2 en Adultos de 30 a 60 Años de edad en la Comunidad de Isla Aguada, Municipio de Ciudad del Carmen, Campeche. RIDE Revista Iberoamericana para la Investigación y el Desarrollo Educativo, vol. 6, núm. 12, enero-junio, 2016 Centro de Estudios e Investigaciones para el Desarrollo Docente A.C. Guadalajara, México. https://www.redalyc.org/articulo.oa?id=498153966027

Tamayo, M. Besoaín, Á. Rebolledo, J. (2018). Determinantes sociales de la salud y discapacidad: actualizando el modelo de determinación. Gac Sanit vol.32 no.1 Barcelona ene./feb. 2018. http://scielo.isciii.es/scielo.php?script=sci_arttext\&pid=S0213-91112018000100096 
Valdés, W. Almirall, A. Gutiérrez, M. (2019). Factores de riesgo de diabetes mellitus tipo 2 en adolescentes. MediSur, vol. 17 núm.

2019. https://www.redalyc.org/jatsRepo/1800/180062367010/html/index.html

Vásquez, Y. Macías, P. González, A. Pérez, C. Carrillo, C. (2019). Determinantes de la salud en adolescentes de la comunidad Cerro Guayabal, Ecuador. Investigación. Rev Cubana Salud Pública 45 (2) 15 Jul 2019Apr-Jun 2019. https://scielosp.org/article/rcsp/2019.v45n2/e1451/

Vidal, D. Chamblas, I. Zavala, M. Müller, R. Cecilia, M. Chávez, A. (2014). Determinantes sociales en salud y estilos de vida en población adulta de Concepción, Chile. Cienc. enferm. vol.20 no.1 Concepción abr. 2014. https://scielo.conicyt.cl/scielo.php?script=sci_arttext\&pid=S0717-95532014000100006 